\title{
Tricofagia en Chinchillas (Chinchilla lanígera): Un problema de Bienestar Animal.
}

\author{
Tamara A. Tadich ${ }^{1}$, Valeria Franchi, Diana Navarrete. \\ ${ }^{1}$ Departamento de Fomento de la Producción Animal, Facultad de Ciencias Veterinarias y Pecuarias. \\ Universidad de Chile, Santa Rosa 11735, La Pintana, Santiago. \\ Email: tamaratadich@u.uchile.cl
}

\begin{abstract}
Resumen
Las estereotipias son desórdenes conductuales que se desarrollan en los animales como consecuencia de ambientes sub-óptimos, sobre los cuales no tienen control. Al estar asociadas con problemas de manejo que afectan el bienestar de los animales, y muchas veces resultar en un deterioro del fitness del animal estas pueden ser utilizadas como un indicador directo (basado en el animal) de bienestar animal. En el caso de la chinchilla la conducta de tricofagia (chinchillas come-pelo) ha sido asociada con problemas de manejo cuando estas son mantenidas en criaderos con fines comerciales y algunos factores individuales. A pesar de que se le ha dado menos atención en los estudios de estereotipias a la chinchilla, es importante su estudio ya que aún existe un importante número de criaderos de esta especie con fines peleteros, y la conducta de tricofagia no sólo afecta el bienestar del individuo, si no que además afecta su producto principal, la piel, por lo que es una patología conductual de importancia económica para el productor. El objetivo de la presente revisión es dar a conocer algunos posibles factores causales de la conducta de tricofagia en chinchillas y las implicancias de las estereotipias sobre el bienestar animal.
\end{abstract}

Palabras clave: estereotipia, bienestar animal, chinchilla (Chinchilla lanígera), tricofagia. 


\section{Introducción}

El bienestar animal es un área de investigación de gran relevancia en la actualidad, encontrándose en la literatura una gran variedad de estudios que evalúan el estado de bienestar de distintas especies a través del uso de indicadores de tipo directo e indirecto, cobrando cada vez mayor importancia aquellos indicadores basados en el animal (directos), como la conducta.

\section{Bienestar Animal}

Broom (1986) define el bienestar animal como "el estado de un animal en relación a sus intentos por sobrellevar su medio ambiente": El animal puede adaptarse de manera exitosa, necesitar de un mayor esfuerzo para adaptarse o simplemente fallar en sus intentos de adaptación, lo cual puede incluso llevar a la muerte del individuo. Esta definición de bienestar animal tiene varias implicancias, entre ellas el ser una característica inherente al animal y no algo que se le entrega, el poder variar de muy bueno a muy pobre y poder ser medible de manera científica e independiente a consideraciones de tipo moral (Broom, 1991).

A través de una perspectiva un poco diferente Duncan y Fraser (1997) consideran el concepto de bienestar animal basado en tres aspectos fundamentales: 1) las experiencias del animal, tales como placer o dolor; 2) el funcionamiento biológico normal del individuo, y 3) la naturalidad de la especie (telos), asegurando que el animal pueda realizar el repertorio conductual de su especie. Esta definición parece ser más completa, ya que toma en cuenta los aspectos físicos, mentales y conductuales del individuo; además, se encuentra en armonía con la definición propuesta por Dawkins (2008), quien establece que se debiera poner énfasis en un estado de salud adecuado y que el animal pueda obtener lo que quiere (concepto de necesidades), entregando así una definición más amigable del concepto que puede ser entendida no sólo en el ambiente científico sino también por el público en general.

\subsection{Desórdenes Conductuales Como Indicador de Bienestar Animal}

Dentro de los indicadores de bienestar animal posibles de utilizar, cobran cada vez más importancia aquellos directos de tipo conductual y, dentro de éstos, la presentación de desviaciones conductuales, particularmente por las implicancias físicas, fisiológicas y mentales que este tipo de conducta trae consigo. Existe un gran número de publicaciones que entregan antecedentes sobre las diversas desviaciones conductuales que se pueden observar en los animales, muchas veces haciendo confusa su comprensión y clasificación (Tadich y Araya, 2010). Muchas veces algunas conductas son inadecuadamente catalogadas como "anormales", debido a una falta de conocimiento del repertorio de conductas normales que presenta el animal o porque representan una molestia para el propietario. Según Broom y Fraser (2007), las anormalidades tienden a ocurrir cuando la frecuencia de los movimientos, intensidad de las acciones o contexto en el cual ocurre la conducta son diferentes a lo normal. En ciertas ocasiones estas conductas no deseadas parecen tener una función adaptativa para el animal, permitiéndole de esta manera enfrentar el cautiverio (Boyd, 1986). En términos generales las conductas no deseadas tienden a reducir el fitness del individuo, el valor del animal o ambos (Fraser, 1992). Por otra parte la mayoría de estas conductas son consecuencia o causa de un deterioro del bienestar del individuo, por lo tanto, ellas debieran tratar de prevenirse en la medida que sea posible, ya que una vez que se instalan en el repertorio conductual del animal son muy difíciles de revertir (Boyd, 1986).

Además del sufrimiento mental que pueden presentar los animales con conductas no deseadas, se han mencionado otras consecuencias físicas $\mathrm{y}$ fisiológicas que acompañan a estas conductas y que también tienen implicancias sobre el estado de bienestar. Entre ellas se puede mencionar la pérdida de condición corporal, problemas de salud asociados, dolor crónico, automutilaciones, y elevación de los niveles de glucocorticoides entre otros (Bhatnagar et al., 2006). Todos estos problemas impiden de una forma u otra que el animal se pueda adaptar de forma adecuada a su medio ambiente y pueden ser evaluadas y medidas de manera objetiva y científica.

Dentro de las desviaciones conductuales las estereotipias probablemente las mayormente estudiadas. Mason (1991) describe las conductas estereotipadas como patrones de comportamiento invariantes y repetitivos, que no tienen una meta o función obvia. Los estereotipos pueden generarse en aquellos animales enfrentados en su medio ambiente a problemas sin solución (como el encierro en una jaula) (Waters et al., 2002). Hay que tener en cuenta otras causales de este tipo de conductas, tales como daño cerebral, condiciones psiquiátricas, o efecto de alguna droga como la d-anfetamina (Robbins, 1976). 
En el caso de las personas, las conductas estereotipadas han sido relacionadas con psicopatologías, siendo éstas un componente característico de la esquizofrenia y el autismo temprano (Dantzer, 1986). Es por esto que, previo a diagnosticar una conducta como estereotipada, se hace esencial la exclusión de cualquier factor de tipo patológico, que pudiese originar un cambio conductual en el animal (Mills et al., 2005). Las primeras descripciones de estereotipias se realizaron en equinos y en animales silvestres en cautiverio en la década de 1960 y, luego en animales de granja mantenidos en sistemas altamente intensivos de producción (Dantzer, 1986). En años recientes se ha estimado que las conductas estereotipadas son realizadas por cerca de 85 millones de animales, incluyendo de granja, laboratorio y zoológico (Mason y Latham, 2004).

Muchas veces debido a condiciones inadecuadas de manejo ambiental y social, los animales no son capaces de satisfacer sus necesidades quedando en un estado motivacional muy alto, lo que puede traer consecuencias como la aparición de conductas no deseadas. Esto causa, además, un estado de ansiedad y frustración crónica, con lo cual se afecta su estado de bienestar. Wiepkema y Koolhaas (1993) indican que las estereotipias son conductas indicativas de un problema de bienestar pasado o presente en el animal. Algunos autores (Broom y Johnson, 1993) sugieren que cualquier nivel de estereotipia representa un problema de bienestar, mientras que otros describen una relación inversa donde a mayor nivel de estereotipia menor estado de bienestar (Dawkins, 1990), y por último otros han propuesto ciertos porcentajes máximos aceptables como 5\% (Wiepkema et al., 1983) o 10\% (Broom, 1991) de individuos con estereotipias dentro de una población. $\mathrm{Si}$ se toma en cuenta que el bienestar animal es un estado del individuo, basta con que un animal presente un trastorno conductual para pensar que este presentó o presenta un problema de bienestar.

La preocupación por las conductas estereotipadas tiene relación con el efecto deletéreo que estas pueden tener sobre el animal y su rendimiento, ya sea en áreas como la reproducción, producción o salud. Se ha podido establecer que al menos un $68 \%$ de las situaciones que favorecen la presentación de estereotipias son también causales de un deficiente bienestar (Mason y Latham, 2004), y pueden ser consideradas como indicador de éste (Parker et al., 2008). Para poder utilizar correctamente la estereotipia como indicador de pobre bienestar hay que tener claridad en el mecanismo de su desarrollo. Mason y Latham (2004) sugieren que en aquellos casos en que la conducta ha pasado a ser un hábito controlado a nivel central o cuando posee características autísticas en el control de ésta, posiblemente no será un buen indicador de bienestar, ya que pueden persistir a pesar de realizarse cambios adecuados en el ambiente del animal.

\subsection{Estereotipias en Roedores}

La manera en que se presente una conducta estereotipada depende de la especie y, dentro de cada especie, puede existir variaciones entre individuos, especialmente en lo que se refiere a repetibilidad y flexibilidad del patrón conductual (Mason, 1991). Por otra parte, el contexto en que se desarrollan las estereotipias está generalmente ligado de manera física y temporal a ambientes sub-óptimos, los que generan situaciones de frustración, miedo o estrés, restricción física y falta de estimulación (Mason, 1991). Dentro de los factores físicos-ambientales, se encuentran por ejemplo la restricción de la alimentación y de la disponibilidad de uso del tiempo de los individuos (Kiley-Worthington, 1987). Es así como en el caso de los roedores la mayoría de ellos desarrolla estereotipias cuando son mantenidos en jaulas sin estímulos ambientales, como las comúnmente utilizadas en experimentación biomédica (Würbel, 2006). A pesar de que los factores ambientales parecen jugar un rol importante en la presentación de estereotipias, no todos los animales mantenidos dentro de un mismo sistema llegan a presentarlas (Mills y Riezebos, 2005).

Existen diferencias entre especies de roedores en cuanto a la presentación de estas conductas, es así como aproximadamente un 50\% de los ratones de laboratorio desarrollan estereotipias, siendo la conducta más común el morder barras, mientras que sólo existen reportes anecdóticos de su presentación en ratas (Würbel y Stauffacher, 1994). Así también las estereotipias parecen ser frecuentes en los gerbos (Meriones unguiculatus) (Wiedenmayer, 1997), el topillo rojo (Clethrionomys glareolus) (Ödberg, 1986) y en chinchillas (Chinchilla lanígera) (Kersten, 1997); mientras que son menos frecuentes en los cuy (Cavia porcellus) (Würbel, 2006). 
Las diferencias en la presentación de estereotipias entre especies podría estar relacionada con otras posibles causales de estereotipias que se han sugerido en la literatura, como la heredabilidad (Vecchiotti y Galanti, 1986) y el temperamento. A pesar de no existir aún evidencia de que estas conductas son heredables, se podría heredar la tendencia o predisposición a realizar una estereotipia y no la conducta en si, la cual sólo se haría evidente bajo ciertos factores de riesgo presentes en el ambiente del animal (Kiley-Worthington, 1987; Mills et al., 2005). Por otra parte se ha propuesto una relación entre temperamento y estereotipias, siendo aquellos animales de temperamento reactivo mayormente predispuestos a realizar estas conductas (Kiley-Worthington, 1987).

Se han mencionado también como posibles factores causales de estereotipias el aprendizaje social y observacional, aunque no existe evidencia que avale dicha teoría (Murphy y Arkins, 2007). En el caso que las conductas estereotipadas funcionarían, a lo menos en parte, como un mecanismo para satisfacer ciertas necesidades que se ven frustradas dentro de un ambiente subóptimo o como estrategia para reducir los niveles de ansiedad, entonces podría ser beneficioso para el animal aprender a realizar estas conductas a través de la observación de individuos más experimentados (Nicol, 1999); y en términos generales su nivel de bienestar podría entonces verse aumentado (Würbel, 2006). Esto más bien podría tratarse de un fenómeno de facilitación social, donde se hace evidente una conducta ya establecida en el animal ante la presencia de otro animal que se encuentre realizándola (Mills et al., 2005). La mayoría de los estudios se han enfocado en la asociación de algunos factores de manejo y la presentación de conductas estereotipadas, dejando de forma secundaria la relación con factores biológicos. Por otra parte, McBride y Hemmings (2009) sugieren que las estereotipias son el resultado de cambios neurológicos como respuesta a un estrés crónico.

\subsection{El Caso de la Chinchilla,}

En el caso particular de la chinchilla (Chinchilla lanígera) se trata de un roedor histricomorfo sudamericano que posee una de las pieles más valiosas en el mundo. Es por esto que ha sido domesticada, criada y seleccionada de acuerdo a su calidad de piel, color y tasa de crecimiento (Grau, 1986). Una de las consecuencias del manejo en cautiverio de las chinchillas con fines comerciales, es la aparición de una conducta no deseada conocida como tricofagia, come pelo, o fur-chewing (Ponzio et al., 2007). En este desorden conductual, clasificado dentro del grupo de las automutilaciones, el animal mastica su propio pelo, el de un compañero, o ambos; es una conducta que se presenta comúnmente en animales domésticos como el visón, conejos, hámster entre otros y a veces puede ocurrir en animales silvestres como el zorro (Rees, 1962). En las chinchillas se puede observar un área del pelaje con las fibras de pelo quebradas por los dientes del animal, como generalmente el animal ataca su propio pelaje, es posible ver zonas desprolijas de pelo en todo el cuerpo menos la cabeza (Rees, 1962) (Figura 1 a y b).

Figura 1. Chinchillas de 11 meses con áreas de pelo hirsuto debido a la conducta de tricofagia en zonas laterales y de extremidades posteriores.

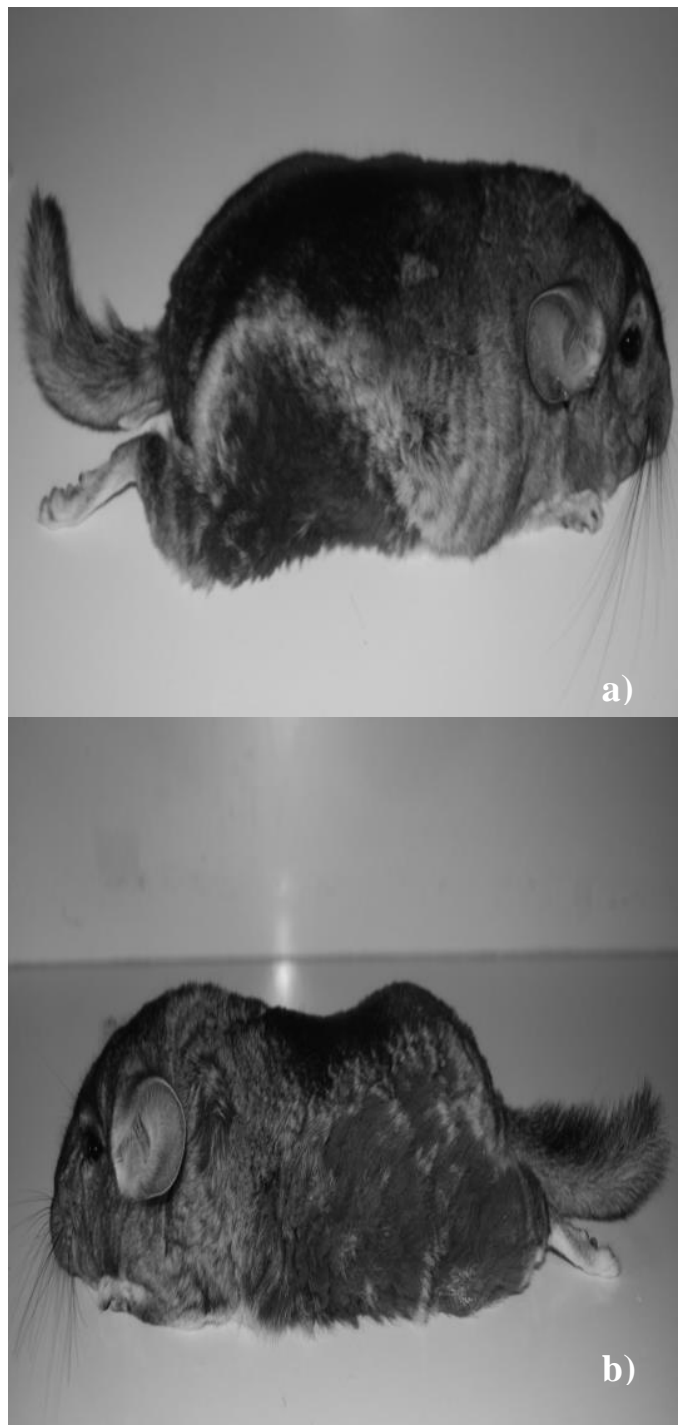


Algunos autores describen frecuencias de presentación de tricofagia de hasta un $30 \%$ de los individuos (Rees, 1962), mientras que estudios en Chile han detectado una presentación en aproximadamente un $4 \%$ de la población perteneciente a criaderos (Tadich datos sin publicar*) (Figura 2).

Figura 2. Frecuencia de presentación (\%) de Chinchillas con conducta "fur chewing" por año, entre los años 1990 y 2009

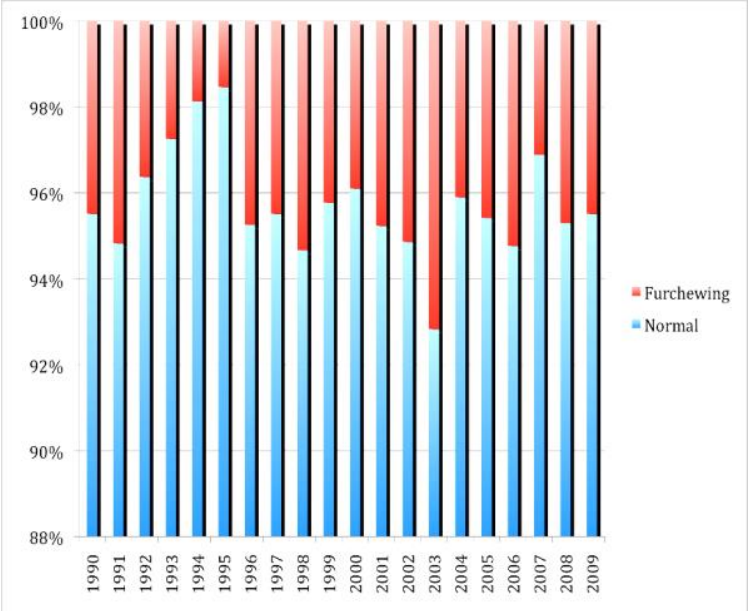

La etiología de esta conducta aún es desconocida y existe muy poca información científica al respecto, sin embargo una serie de hipótesis se han postulado, dentro de ellas destacan: a) un posible problema de malnutrición, b) causas bacteriológicas, fúngicas y parasitológicas, c) estrés relacionado con causas ambientales (Ponzio et al., 2007). Las primeras dos teorías relacionadas con un problema de malnutrición o la presencia de un posible agente patógeno han sido prácticamente descartadas. En el caso de la primera por ejemplo el estudio de Tisljar et al. (2002) mostró que no existía presencia de pelos en el tracto digestivo de los animales que presentaban la conducta, por lo que no se trataría de una tricofagia verdadera, si no que de un acicalamiento excesivo como ocurre en otras especies como es el caso de las aves. Los mismos autores no pudieron evidenciar patógenos significativos que afectaran de manera evidente la salud de la piel de los animales (Tisljar et al., 2002). Por otra parte la presencia de un agente patógeno como agente etiológico de esta conducta descartaría su clasificación como una conducta no deseada estereotipada.
En relación a factores ambientales que pudiesen estar produciendo un estrés crónico en las chinchillas, por ende favoreciendo el desarrollo de la conducta, Ponzio et al. (2007) realizaron un estudio transversal en 101 criaderos de chinchillas con fines comerciales en Argentina. Los autores reportaron factores como el espacio disponible, los ruidos externos y la falta de baños de polvo como posibles factores que podrían favorecer el desarrollo de esta conducta. Otros factores ambientales que se han asociado a la presentación de la conducta incluyen ruidos constantes por cercanía a autopistas de los criaderos, dietas inapropiadas, jaulas en malas condiciones de limpieza y condiciones de microambiente inadecuadas como temperaturas $\mathrm{y}$ humedades distintas a $12^{\circ} \mathrm{C}$ y $60 \%$ respectivamente (Mösslacher, 1986; Grauvogl, 1990; Merry, 1990; Jenkins, 1992).

Por otra parte en el caso de los roedores existen antecedentes experimentales de cambios patológicos en la glándula adrenal, de hiperplasia e hipertrofia, asociados a zonas específicas de la corteza y médula cuando estas son sometidas a un estrés crónico, además de cambios relacionados con peso corporal, temperatura corporal (Bhatnagar et al., 2006; Ulrich-Lai et al., 2006). En el caso de las chinchillas Vanjonack y Johnson (1973) demostraron actividad aumentada de la tiroides y de las hormonas adrenocorticales en chinchillas que presentaban tricofagia. Así mismo Tisljar et al. (2002) reportaron hiperplasia adrenal en todas las chinchillas come pelo estudiadas. En un estudio más reciente Ponzio et al. (2012) reportaron cambios hormonales relacionados con una respuesta de estrés en las chinchillas que presentaban la conducta, a través de la medición de la concentración de metabolitos de glucocorticoides en orina y un incremento de conductas relacionadas con un estado de ansiedad aumentado en los animales sometidos a una prueba de laberinto, cambios que fueron más evidentes en las hembras. Siendo la corticoesterona la hormona glucocorticoide de preferencia a estudiar cuando se evalúa la respuesta de estrés en la chinchilla (Ponzio et al., 2004; Palme et al., 2005). Estos estudios suman evidencia de que la tricofagia sería un síndrome hereditario de naturaleza congénita o conductual donde los animales afectados podrían ser variantes más sensibles al estrés, que no logran sobrellevar el estrés del cautiverio (Tisljar et al., 2002), quedando en mayor riesgo de presentar problemas asociados a un pobre bienestar animal. 


\section{Implicancias para el Bienestar Animal}

Al enfrentarnos con un animal que presenta una desviación conductual hay que tener en cuenta que cualquiera sea el origen de ésta (ambiental, social, patológica, etc.), su estado de bienestar se encuentra reducido, ya sea por la causa que genera su presentación, por las consecuencias de su realización o las posibles medidas de control o tratamiento que se adopten (Tadich y Araya, 2010). En el caso de la tricofagia en chinchillas destinadas a peletería, además de afectar el bienestar del individuo la realización de la conducta tiene consecuencias económicas para el productor.

Mientras se mantenga la industria peletera, la implementación de protocolos de evaluación de bienestar animal, diseñados especialmente para este tipo de industria en Europa como el proyecto WelFur (B) son importantes de implementar para asegurar estándares mínimos de bienestar animal. Es importante destacar que este proyecto fue desarrollado especialmente para zorros y visones, pero podría ser extrapolado a la chinchilla, otra consideración a tener en cuenta es que el protocolo contempla un gran número de indicadores basados en el animal (directos) y dentro de ellos la posibilidad de los animales de poder desarrollar conductas apropiadas para su especie, siendo la presentación de la tricofagia un indicador de pobre bienestar (Mononen et al., 2012).

La identificación de estas conductas por parte del propietario y del médico veterinario, y la correcta asociación de su presentación con factores de riesgos presentes en el sistema permitirán el desarrollo de prácticas de manejo y diseños de recintos adecuados para la mantención de animales que consideren ambientes más complejos, donde el individuo pueda tener un mayor control de éste, por ejemplo a través de la inclusión de enriquecimiento ambiental, evitándose la aparición de estas conductas en el futuro.

Agradecimientos: Al proyecto de investigación FIV DI-FAVET 2012,

\section{Referencias}

1. Bhatnagar, S.; Vining, C.; Iyer, V.; Kinni, V. 2006. Changes in the hypothalamic-pituitaryadrenal function, body temperature, body weight and food intake with repeated social stress exposure in rats. J. Neuroendocrinol. $18,13-24$.

2. Boyd, L.E. 1986. Behavior problems of equids in zoos. Vet. Clin. N. Am. Equine Pract. 2, 653-664.

3. Broom, D.M. 1986. Indicators of poor welfare. Br. Vet. J. 142, 524.

4. Broom, D.M. 1991. Animal welfare: concepts and measurements. J. Anim. Sci. 69, 4167-4175.

5. Broom, D.M.; Johnson, K. 1993. Stress and animal welfare. Chapman and Hall, London, UK.

6. Broom, D.M.; Fraser, A.F. 2007. Abnormal behaviour 1: stereotypies. In: Broom DM, Fraser AF (eds). Domestic animal behaviour and welfare. 4th ed. Oxford University Press, New York, USA, Pp 226-234.

7. Canali, E.; Borroni, A. 1994. Behavioural problems in thoroughbred horses reared in Italy. Appl. Anim. Behav. Sci. 40, 74.

8. Dantzer, R. 1986. Behavioral, physiological and functional aspects of stereotyped behavior: a review and a re-interpretation. $J$. Anim. Sci. 62, 1776-1786.

9. Dawkins, M.S. 1990. From an animals point of view: motivation, fitness and animal welfare. Behav. Brain Sci. 13, 1-61.

10. Dawkins, M.S. 2008. The science of animal suffering. Ethology 114, 937-945.

11. Duncan, I.J.H.; Fraser, D. 1997. Understanding animal welfare. In: Appleby MC, Hughes BO (eds). Animal welfare. CABI, Wallingford, UK, Pp 19-31. 
12. Fraser A. 1992. Abnormal and anomalous behaviour. In: Fraser A (ed). The behaviour of the horse. CABI, Newfoundland, Canada.

13. Grau, J. 1986. La chinchilla, su crianza en todos los climas. 3era edición. El Ateneo, Buenos Aires, Argentina.

14. Grauvogl, A. 1990. Raising of für-bearing animals and animal welfare. Dtsch. Tierärztl. Wochenschr. 97, 164-167.

15. Jenkins, J.R. 1992. Husbandry and common diseases of the chinchilla (C. Laniger). J. Small Exot. Anim. Med. 2, 15-17.

16. Kersten, A.M.P. 1997. Behaviour and welfare of chinchillas in commercial farming: a preliminary study. In: Hemsworth, P.H.; Spinka, M.; Kostal, L. (eds). Proceedings of the 31st international society for applied ethology. The International Society of Applied Ethology, Prague, p.171.

17. Kiley- Worthington, M. 1987. Behavioural problems. In: Kiley-Worthington M (ed). The behaviour of horses in relation to management and training. J. A. Allen, London, UK.

18. Mason, G. 1991. Stereotypies - a critical review. Anim. Behav. 41, 1015-1037.

19. Mason, G.J.; Latham, N.R. 2004. Can't stop, won't stop: is stereotypy a reliable animal welfare indicator?. Anim. Welf. 13 Suppl 1, 57-69.

20. Merry, C.J. 1990. An introduction to chinchillas. Vet. Tech. 11, 315-322

21. McBride, S.D; Hemmings, A. 2009. A neurologic perspective of equine stereotypy. J. Equine Vet. Sci. 29, 10-16.

22. Mills, D.S.; Riezebos, M. 2005. The role of the image of a conspecific in the regulation of stereotypic head movements in the horse. Appl. Anim. Behav. Sci. 91, 155-165.

23. Mills, D.A.; Taylor, K.D.; Cooper, J.J. 2005. Weaving, Headshaking, Cribbing and other stereotypies. In Depth: Behavior. AAEP

Proceedings, vol 51. Washington, USA.

24. Mononen, J.; Moller, S.H.; Hansen, S.W.; Hovland, A.L.; Koistinen, T.; Lidfors, L.; Malmkvist, J.; Vinke, C.M.; Ahola, L. 2012. The development of on-farm welfare assessment protocols for foxes and mink: the WelFur project. Anim. Welf. 21, 363-371.

25. Mösslacher, E. 1986. Für biting. In: Mösslacher, E. (ed) Breeding and caring for chinchillas. T.F.H. Publ., Neptune City. Pp.82-91.

26. Murphy, J.; Arkins, S. 2007. Equine learning behaviour. Behav. Process. 76, 1-13.

27. Nicol, C.J. 1999. Understanding equine stereotypies. Equine. Vet. J, suppl. 28, 20-25.

28. Ödberg, F.O. 1986. The jumping stereotypy in the bank vole (Clethrionomys glareolus). Biology of Behav. 11, 130-143.

29. Parker, M.; Redhead, E.S.; Goodwin, D.; McBride, S.D. 2008. Impaired instrumental choice in crib biting horses (Equus caballus). Behav. Brain. Res. 191, 137-140.

30. Palme, R.; Rettenbacher, S.; Touma, C.; ElBahr, S.M.; Möstl, E. 2005. Stress hormones in mammals and birds. Comparative aspects regarding metabolism, excretion and noninvasive measurement in fecal samples. Ann. N. Y. Acad. Sci. 1040, 162-171.

31. Ponzio, M.F.; Monfort, S.L.; Busso, J.M.; Dabbene, V.G.; Ruiz, R.D.; Fiol de Cuneo, M. 2004. A non-invasive method for assessing adrenal activity in the chinchilla (Chinchilla lanígera). Journal of Experimental Zoology 301A 218-227.

32. Ponzio, M.F.; Busso, J.M.; Ruiz, R.D.; Fiol de Cuneo, M. 2007. A survey assessment of the incidence of fur-chewing in commercial chinchilla (Chinchilla lanígera) farms. Anim. Welf. 16, 471-479.

33. Ponzio, M.F.; Monfort, S.L.; Busso, J.M.; Carlini, V.P.; Ruiz, R.D.; Fiol de Cuneo, M. 2012. Adrenal activity and anxiety-like behavior in fur-chewing chinchillas 
(Chinchilla lanígera). Horm. Behav. 61, 758762.

34. Rees, R.G. 1962. Some conditions of the skin and fur of Chinchilla lanígera. J. Small Anim. Pract. 4, 213-225.

35. Tadich, T.; Araya, O. 2010. Conductas no deseadas en equinos. Arch. Med. Vet. 42, 2941.

36. Tisljar, M.; Janic, D.; Grabarevic, Z.; Marinculic, A.; Pinter, L.; Janicki, Z.; Nemanic, A. 2002. Stress induced cushing's syndrome in fur chewing chinchillas. Acta. Vet. Hung. 50, 133-142.

37. Ulrich-Lai, Y.M.; Figueiredo, H.F.; Ostrander, M.M.; Choi, D.C.; Engeland, W.C.; Herman, J.P. 2006. Chronic stress induces adrenal hyperplasia and hypertrophy in a subregion-specific manner. Am. J. Physiol. Endocrinol. Metab. 291, E965E973.

38. Vanjonack, W.J.; Johnson, H.D. 1973. Relationship of thyrod and adrenal function to "fur-chewing" in the chinchilla. Comp. Biochem. Physiol. 45A, 115-120.

39. Vecchiotti, G.G.; Galanti, R. 1986. Evidence of heredity of cribbing, weaving and stall walking in thoroughbred horses. Livest. Prod. Sci. 14, 91-95.

40. Waters, A.J.; Nicol, C.J.; French, N.P. 2002. Factors influencing the development of stereotypic and redirected behaviours in young horses: findings of a four year prospective epidemiological study. Equine. Vet. J. 34, 572-579.

41. Wiedenmayer, C. 1997. Causation of the ontogenetic development of stereotypic digging in gerbils. Anim. Behav. 53, 461470 .

42. Wiepkema, P.R.; Broom, D.M.; Duncan, I.; van Putten, G. 1983. Abnormal behaviour in farm animals - Report of the commission of the European communities. Commission of the European Communities, Brussels, Belgium.
43. Wiepkema, P.R.; Koolhaas, J.M. 1993. Stress and animal welfare. Anim. Welf. 2, 195-218.

44. Würbel, H. 2006. The motivational basis of caged rodents' stereotypies. In: Mason, G.; Rushen, J. Stereotypic Animal Behaviour, Fundamentals and applications to welfare. $2^{\text {nd }}$ Ed. CABI, London, UK.

45. Würbel, H., Stauffacher, M. 1994. StandardHaltung für labormäuse - Probleme und lösungsansätze. Tierlaboratorium 17, 109118. 